\title{
Phase I Trial
}

National Cancer Institute

\section{Source}

National Cancer Institute. Phase I Trial. NCI Thesaurus. Code C15600.

A clinical research protocol designed to test a new biomedical intervention in a small group of people for the first time. A Phase I trial can be to establish the toxicity of a new treatment with escalating intensity of the treatment administered and/or to determine the side effects of a new treatment for a particular indication in subjects. 